\title{
Human Activity Recognition with 2D and 3D Cameras
}

\author{
Zicheng Liu \\ Microsoft Research \\ One Microsoft Way \\ Redmond WA 98052 \\ zliu@microsoft.com
}

\begin{abstract}
This presentation will cover human activity recognition by using conventional $2 \mathrm{D}$ video cameras as well as the recently developed 3D depth cameras. I'll first give an overview on the interest- point based approach which has become a popular research direction in the past few years for $2 \mathrm{D}$ based activity recognition. In addition to the conventional classification problem, I'll discuss the problem of detection (spacetime localization) as well as the example-based search where the amount of labelled data is extremely small. The second part of the talk will focus on activity recognition with 3D depth cameras. I'll describe some of the recently developed visual representations and machine learning frameworks for 3D data analysis.
\end{abstract}

